\title{
Consumo de pornografía y malestar asociado: diferencias entre hombres y mujeres
}

\author{
Juan Enrique Nebot-Garcia \\ junebot@uji.es \\ Marcel Elipe-Miravet \\ al226029@uji.es \\ Marta García-Barba \\ barbam@uji.es \\ Ana Antelo-García \\ al386592@uji.es \\ Rafael Ballester-Arnal \\ rballest@uji.es
}

\section{Resumen}

Introducción: La pornografía puede contribuir al desarrollo sexual de los jóvenes, pero también puede constituir un factor facilitador de insatisfacción sexual, dados los falsos modelos que representa.

Metodología: 250 hombres y 250 mujeres, con 21,1 años de media $(D T=1,56)$, cumplimentaron un cuestionario en línea sobre visionado de pornografía. El $72,2 \%$ eran heterosexuales y el $27,8 \%$ no heterosexuales.

Resultados: El $68 \%$ de participantes ha visto porno gay; el $81,8 \%$, lésbico y el $92 \%$, heterosexual. Por lo que respecta a la excitación, entre aquellos que han visionado cada tipo de material, un $45,9 \%$ de hombres y un $41,8 \%$ de mujeres se han excitado con porno gay, siendo un $25,8 \%$ de hombres y un $6,6 \%$ de mujeres los que sintieron malestar por haberse excitado. Con el lésbico se han excitado un $78,3 \%$ de hombres y un $71,5 \%$ de mujeres, y un $4,2 \%$ de mujeres y ningún hombre ha sentido malestar por ello. Por último, con el heterosexual, se han excitado un $93,9 \%$ de hombres y un $94 \%$ de mujeres, y un $1,3 \%$ de hombres y un $4,9 \%$ de mujeres han experimentado malestar ante su excitación. Se han observado diferencias de género significativas en los distintos porcentajes de visionado y de malestar, pero no en los de excitación.

Conclusiones: El género parece ser un factor diferencial en el consumo de pornografía, así como del malestar asociado. Por tanto, debería profundizarse en su papel, así como tenerse en cuenta a la hora de elaborar programas de educación sexual adecuados para un uso saludable de la pornografía.

Pa labras clave: pornografía, excitación, malestar, género. 


\section{Abstract}

Introduction: Pornography can contribute to the sexual development of young people but it can also be a facilitator of sexual dissatisfaction, given the false models it represents.

Methodology: 250 men and 250 women, with an average age of 21.11 years $(\mathrm{SD}=1.56)$, carried out an online questionnaire about viewing pornography. $72.2 \%$ were heterosexual and $27.8 \%$ non-heterosexual.

Results: $68 \%$ of participants have seen gay porn, $81.8 \%$ lesbian and $92 \%$ heterosexual. According to the excitement, among those who have viewed each type of material, $45.9 \%$ of men and $41.8 \%$ of women have been excited by gay porn, and $25.8 \%$ of men and $6.6 \%$ of women have felt discomfort for being aroused. With the lesbian, $78.3 \%$ of men and $71.5 \%$ of women have been excited, and $4.2 \%$ of women and no man have felt discomfort for that. Finally, with the heterosexual, $93.9 \%$ of men and $94 \%$ of women have been excited, and $1.3 \%$ of men and $4.9 \%$ of women have experienced discomfort with their excitement. Significant gender differences have been observed in the different percentages of viewing and discomfort, but not in those of excitation.

Conclusions: Gender seems to be a differential factor in the consumption of pornography, as well as the associated discomfort. Therefore, its analysis should be deepened, as well as taken into account when developing appropriate sex education programs for the healthy use of pornography.

Keywords: pornography, excitement, discomfort, gender.

\section{Introducción}

El consumo de pornografía puede ser utilizado como una fuente de información y servir de recurso educativo para jóvenes (Ševčíková y Daneback 2014), además de contribuir a un desarrollo satisfactorio de la sexualidad, ya que se ha observado que aquellas personas que visionan material erótico refieren disfrutar de experiencias sexuales más satisfactorias y tienen mayor apertura sexual (Rissel et al. 2017). No obstante, un consumo excesivo y regular puede afectar a la calidad de vida sexual (Poulsen, Busby y Galovan 2013; Wright, Miezan y Sun 2019), así como provocar discusiones en la pareja (Willoughby, Carroll, Busby y Brown 2016) y favorecer disfunciones sexuales (Park et al. 2016). Además, tener como únicos modelos sexuales a los actores que aparecen en la pornografía puede generar unas falsas expectativas, en cuanto al físico y al desempeño sexual, que pueden afectar a la autoestima y a la propia imagen corporal (Tylka 2015). Eso por no hablar de la imagen lamentable y sexista de la mujer que se transmite en la pornografía que, en la mayoría de los casos, queda reducida a un mero objeto al servicio del placer del hombre.

No obstante, pese a estos efectos adversos, Internet también nos proporciona un espacio donde poder explorar nuestra sexualidad de forma más segura que en la vida real (Griffiths 2012). Además, existe un amplio abanico de materiales eróticos, de diferentes tipologías, prácticas... que pueden servirnos para satisfacer nuestra curiosidad, explorar nuevos horizontes y disfrutar de experiencias sexuales que no podemos realizar en nuestro día a día, como pueden ser el visionado de videos sobre ciertos fetiches y fantasías (Yu 2013) o de prácticas sexuales propias de otra orientación sexual (Hald y Štulhofer 2016; Walters y Spengler 2016). Sin embargo, esta exploración también puede llevar asociada un sentimiento de malestar, sobre 
todo cuando una persona no esperaba excitarse con un tipo de material en concreto y se produce una disonancia con su propio autoconcepto (Priolo et al. 2016), como es el caso de materiales de otras orientaciones sexuales (Walters y Spengler 2016).

Esta vivencia de la sexualidad y de la pornografia difiere entre mujeres y hombres. Al igual que ocurre con la sexualidad en general, donde de una manera sexista se espera que los hombres sean más activos y muestren mayor interés por el sexo (Emmerink, Vanwesenbeeck, van den Eijnden y ter Bogt 2016), los hombres también presentan mayor actividad en el visionado de pornografía. De hecho, pese a que las mujeres también refieren visualizar pornografía, los hombres recurren a ella con mayor frecuencia que las mujeres (Ballester-Arnal, Castro Calvo, Gil-Llario y Gil-Juliá 2017; Castro-Calvo, García-Barba, Gil-Juliá, Morell-Mengual y Ballester-Arnal 2018; Poulsen, Busby y Galovan 2013).

Este estudio se realiza con la intención de explorar más detalladamente las diferencias entre hombres y mujeres en el consumo de pornografía y el malestar que puede ocasionar, sobre todo cuando la excitación se experimenta ante un material en principio contrario a la propia orientación sexual.

A partir de la literatura científica, se plantean las siguientes hipótesis: 1) los chicos mostrarán mayores prevalencias de visionado en los tres tipos de material erótico (hetero, gay y lésbico); 2) los chicos mostrarán mayores prevalencias de excitación en los tres tipos de material erótico; 3) los hombres mostrarán mayor malestar por haberse excitado con pornografía gay y las mujeres por haberse excitado con el lésbico.

\section{Metodología}

\section{Participantes}

La muestra final constó de 250 hombres y 250 mujeres, de entre 18 y 25 años $(M=21,11 ; D T=1,56)$. Todos ellos eran de nacionalidad española y procedían de diferentes provincias españolas. En cuanto a su orientación sexual, el $72,2 \%$ eran heterosexuales, el 11,8\% homosexuales, el $13 \%$ bisexuales, el 0,8\% pansexuales (pueden sentir atracción por cualquier persona, independientemente de su sexo o identidad de género), el $0,8 \%$ demisexuales (solo sienten atracción en determinados casos donde existe un vínculo emocional fuerte) y el $1,4 \%$ restante de otras orientaciones o preferían no etiquetarse. Esta investigación ha sido posible, en parte, gracias a la ayuda predoctoral PREDOC/2017/45 de la Universitat Jaume I.

Por lo que respecta a los datos sociodemográficos (véase la tabla 1), la mayoría eran ateos o agnósticos $(68,4 \%)$, con estudios universitarios $(64,4 \%)$, progresistas $(48,8 \%)$ y procedían de una localidad urbana $(73 \%)$. Para poder participar en el estudio, debían residir en España, entender el español y tener entre 18 y 25 años. 
Tabla 1

Principales características sociodemográficas de la muestra

\begin{tabular}{|c|c|c|c|}
\hline & & $\mathbf{N}$ & $\%$ \\
\hline \multirow[t]{6}{*}{ Nivel de estudios } & Sin estudios & 1 & 0,2 \\
\hline & Primarios & 5 & 1 \\
\hline & Secundarios & 93 & 18,6 \\
\hline & Formación profesional & 55 & 11,0 \\
\hline & Universitarios & 322 & 64,4 \\
\hline & Máster/doctorado & 24 & 4,8 \\
\hline \multirow{4}{*}{$\begin{array}{l}\text { Lugar de residencia durante } \\
\text { infancia/adolescencia }\end{array}$} & Urbana de costa & 185 & 37 \\
\hline & Urbana de interior & 180 & 36 \\
\hline & Rural de costa & 29 & 5,8 \\
\hline & Rural de interior & 106 & 21,2 \\
\hline \multirow[t]{4}{*}{ Ideología política } & Conservador & 43 & 8,6 \\
\hline & Progresista & 244 & 48,8 \\
\hline & Centro & 98 & 19,6 \\
\hline & Indiferente & 115 & 23 \\
\hline \multirow[t]{3}{*}{ Creencias religiosas } & Creyente practicante & 26 & 5,2 \\
\hline & $\begin{array}{l}\text { Creyente no } \\
\text { practicante }\end{array}$ & 132 & 26,4 \\
\hline & Ateo o agnóstico & 342 & 68,4 \\
\hline
\end{tabular}

Instrumentos

Para este estudio se utilizó un cuestionario sobre visionado de pornografía creado ad hoc por el equipo Salusex-Unisexsida de la Universitat Jaume I de Castellón y la Universidad de Valencia. Este instrumento consta de tres escalas con tres ítems cada una $(\alpha=0,80)$ :

- Por una parte, se evalúa si alguna vez han visionado pornografía heterosexual (ítem 1), gay (ítem 2) o lésbica (ítem 3), debiendo contestar con sí o no.

- En segundo lugar, aquellas personas que contestan afirmativamente a alguna de las anteriores preguntas, en función de qué tipo de material habían visualizado, se les pide que digan si alguna vez se han excitado cuando han visto material erótico heterosexual (ítem 4), gay (ítem 5) o lésbico (ítem 6). Estas preguntas también deben ser contestadas con sí o no.

- Por último, si se respondía afirmativamente a alguno de los ítems sobre excitación, se les preguntaba si el hecho de haberse excitado con material heterosexual (ítem 7), gay (ítem 8) o lésbico (ítem 9), les había generado malestar. Estas preguntas podían contestarse con sí o no.

\section{Procedimiento}

Como paso inicial, se elaboró el cuestionario ad hoc y se adaptó una versión en línea para la recogida de respuestas, que se realizó entre los meses de enero y marzo del 
2017. La investigación fue anunciada en redes sociales y grupos de interés con la finalidad de llegar a un amplio abanico de población. Una vez los participantes accedían al enlace del anuncio, entraban a la plataforma del cuestionario, donde se les explicaba en qué consistía el estudio y se les informaba del carácter anónimo, voluntario y confidencial de la investigación $y$, por último, se les pedía el consentimiento informado. La investigación contaba con el permiso de la Comisión Deontológica de la Universitat Jaume I (Castellón, España) y, en todo momento, se siguieron los principios éticos de la Declaración de Helsinki y se tomaron las medidas oportunas que marca la legislación más reciente para cumplir con la protección de datos.

Para finalizar, con los datos recogidos a través de un muestreo aleatorio simple, se seleccionaron aquellos casos que cumplían los criterios de inclusión descritos anteriormente (residir en España, entender el español y tener entre 18 y 25 años) y se procedió a realizar los análisis estadísticos pertinentes.

\section{Análisis estadísticos}

Se realizaron distintos análisis mediante el programa estadístico SPSS versión 25 . En primer lugar, se realizaron anál isis de frecuencias y descriptivos para obtener los datos demográficos de la muestra.

En segundo lugar, para calcular los porcentajes de visionado, excitación y malestar asociado con los diferentes tipos de pornografía, se utilizaron tablas de contingencias $\mathrm{y}$, para analizar las diferencias entre chicos y chicas, se realizaron pruebas chi cuadrado.

\section{Resultados}

En términos generales, se observa que la pornografía heterosexual es la que muestra mayores prevalencias de visionado $(92 \%)$ y excitación $(93,9 \%)$. En cambio, por lo que respecta al malestar, es la excitación con la pornografía gay la que produce mayor malestar (18\%).

Si se atienden las diferencias entre chicos y chicas (véase la tabla 2), se observa que los chicos son los que han visionado más pornografía, independientemente del tipo de material erótico (heterosexual, gay o lésbico), siendo estas diferencias significativas. Por el contrario, los porcentajes de chicos y chicas que se han excitado con los diferentes tipos de pornografía resulta bastante similar, ya que no se encontraron diferencias significativas. Por lo que respecta al malestar, las chicas muestran mayores índices de malestar al haberse excitado con material erótico heterosexual y lésbico, y los hombres, con el gay, siendo todas las diferencias estadísticamente significativas. 
Tabla 2

Porcentaje de chicos y chicas que han visionado, se han excitado y han sentido malestar con pornografía heterosexual, gay y lésbica

\begin{tabular}{cccc} 
& $\begin{array}{c}\text { Chicos } \\
(\%)\end{array}$ & $\begin{array}{c}\text { Chicas } \\
(\%)\end{array}$ & Chi $^{2}(p)$ \\
\hline Porno hetero & & & \\
Visionado & 97,6 & 86,4 & $21,30(<0,001)$ \\
Excitación & 93,9 & 94 & $0,01(0,954)$ \\
Malestar & 1,3 & 4,9 & $4,82(0,028)$ \\
Porno gay & & & \\
Visionado & 77,6 & 58,4 & $21,17(<0,001)$ \\
Excitación & 45,9 & 41,8 & $0,56(0,452)$ \\
Malestar & 25,8 & 6,6 & $9,12(0,003)$ \\
Porno lésbico & & & \\
Visionado & 92 & 71,6 & $34,94(<0,001)$ \\
Excitación & 78,3 & 71,5 & $2,46(0,116)$ \\
Malestar & 0 & 10,2 & $19,08(<0,001)$
\end{tabular}

\section{Conclusiones}

El presente estudio ha tenido el objetivo de analizar las diferencias entre chicas y chicos en el visionado y la excitación de pornografía heterosexual, lésbica y gay, y observar el malestar asociado con la respuesta de excitación.

En este sentido, se observó que el visionado y la excitación de pornografía se produce con distintos tipos de materiales, independientemente de la propia orientación sexual. Así, el material más consumido y con el que más se excita la población en general es el heterosexual, seguido del lésbico y, en menor medida, el gay. Los hombres son los que mayores porcentajes de visionado presentan, independientemente del tipo de material erótico, siendo estas diferencias significativas respecto a las mujeres, tal y como se había observado en otros estudios (Poulsen, Busby y Galovan 2013). No obstante, en cuanto a la excitación, no se encontraron diferencias significativas entre mujeres y hombres. Estos resultados podrían explicarse por la represión sexual a la que han sido sometidas las mujeres (Van Ness, Miller, Negash y Morgan 2017), ya que existe la creencia tradicional de que las mujeres deben tener menor interés sexual que los hombres (Freixas y Luque 2009), por lo que tradicionalmente no se ha concebido que busquen activamente su placer sexual y experimenten con su sexualidad. Por tanto, debido a esta norma social, las mujeres accederían en menor medida a la pornografía, pero, cuando lo hacen, disfrutarían y se excitarían de forma similar a los hombres. Por otra parte, estos porcentajes de visionado y excitación no se corresponden con los de las orientaciones sexuales que refieren los participantes, lo que iría en la línea de otros estudios que afirman que las personas, pese a tener una determinada orientación sexual, pueden manifestar atracción, conductas o interés hacia el sexo no preferente (Copen, Chandra y Febo-Vazquez 2016; Nebot-Garcia, García-Barba, Gil-Juliá, Giménez-García y Ballester-Arnal 2018; Vrangalova y SavinWilliams 2010). 
En cuanto a la experiencia de malestar, los hombres mostraron mayores prevalencias por haberse excitado con pornografía gay y las mujeres, con la lésbica, obteniéndose diferencias significativas en ambas. Este malestar podría deberse a la discrepancia entre lo que esperamos de nosotros mismos y lo que realmente nos sucede (Priolo et al. 2016). También puede estar influyendo la rigidez de las etiquetas sexuales (DavisDelano y Morgan 2016; Davis-Delano, Morgan, Gillard y Davis 2018), por las que se autocastigan todos aquellos comportamientos que se alejan de lo heterosexual. No obstante, las mujeres también puntuaron más alto que los hombres en el malestar sentido con el porno heterosexual, lo que no guardaría relación con la anterior teoría, sino que podría explicarse por la represión sexual de las mujeres de la que se hablaba anteriormente (Van Ness, Miller, Negash y Morgan 2017). Además, también cabría tener en cuenta la posible influencia de las creencias religiosas (Baltazar, Helm, McBride, Hopkins y Stevens 2010; Nelson, Padilla-Walker y Carroll 2010; Poulsen, Busby y Galovan 2013) o los valores morales (Fisher, Montgomery-Graham y Kohut 2019; Grubbs, Perry, Wilt y Reid 2019).

Si bien este estudio aporta información relevante, también cuenta con algunas limitaciones, como la muestra limitada de personas con una orientación sexual diferente a la heterosexual. Para futuros estudios, con la intención de obtener unos datos más concretos y específicos, sería conveniente aumentar el tamaño muestral de este colectivo para poder comparar el consumo, la excitación y el malestar asociado entre las diferentes orientaciones sexuales, ya que tanto la orientación sexual como el género han resultado ser dos variables diferenciales en la vivencia de diversos aspectos de la sexualidad (Gil-Llario, Morell-Mengual, Ballester-Arnal, Giménez-García y Castro-Calvo 2015). Con todo ello, los hallazgos de este estudio muestran la diversidad de experiencias vividas con la pornografía y el papel diferencial del género, además del malestar que puede tener asociado. Todo ello debería ser tenido en cuenta a la hora de elaborar programas de educación sexual que aborden el uso saludable de la pornografía.

\section{Referencias bibliográficas}

Ballester-Arnal, Rafael, Jesús Castro Calvo, María Dolores Gil-Llario y Beatriz GilJuliá. 2017. «Cybersex addiction: a study on Spanish college students». Journal of Sex \& Marital Therapy, 43(6): 567-585.

Baltazar, Alina, Herbert W. Helm Jr., Duane McBride, Gary Hopkins y John V. Stevens Jr. 2010. «Internet pornography use in the context of external and internal religiosity». Journal of Psychology and Theology, 38(1): 32-40.

Castro-Calvo, Jesús, Marta García-Barba, Beatriz Gil-Juliá, Vicente Morell-Mengual, y Rafael Ballester-Arnal. 2018. "Motivos para el consumo de cibersexo y su relación con el grado de severidad». International Journal of Developmental and Educational Psychology. Revista INFAD de Psicología, 1(1): 93-102.

Copen, Casey E., Anjani Chandra e Isaedmarie Febo-Vazquez. 2016. "Sexual behavior, sexual attraction, and sexual orientation among adults aged 18-44 in the United States: Data from the 2011-2013 National Survey of Family Growth». National Health Statistics Reports, 88: 1-14.

Davis-Delano, Laurel R. y Elizabeth M. Morgan. 2016. «Heterosexual identity management: How social context affects heterosexual marking practices». Identity, 16(4): 299-318.

Davis-Delano, Laurel R., Elizabeth M. Morgan, Ann Gillard y Coralynn V. Davis. 2018. "When Heterosexuality is Questioned: Stifling Suspicion Through Public Displays of Heterosexual Identity». Journal of Homosexuality, 65(13): 16831708. 
Emmerink, Peggy M., Ine Vanwesenbeeck, Regina J. van den Eijnden y Tom F. ter Bogt. 2016. «Psychosexual correlates of sexual double standard endorsement in adolescent sexuality». The Journal of Sex Research, 53(3): 286-297.

Fisher, William A., Stephanie Montgomery-Graham y Taylor Kohut. 2019. «Pornography problems due to moral incongruence». Archives of Sexual Behavior, 48(2): 425-429.

Freixas, Anna y Bárbara Luque. 2009. «El secreto mejor guardado: la sexualidad de las mujeres mayores». Revista Política y Sociedad, 46(1-2): 191-203.

Gil-Llario, María Dolores, Vicente Morell-Mengual, Rafael Ballester-Arnal, Cristina Giménez-García y Jesús Castro-Calvo. 2015. "Sexual sensation seeking in Spanish young men and women with different sexual orientations». Journal of Sex \& Marital Therapy, 41(5): 525-530.

Griffiths, Mark D. 2012. "Internet sex addiction: A review of empirical research». Addiction Research and Theory, 20(2): 111-124.

Grubbs, Joshua B., Samuel L. Perry, Joshua A. Wilt y Rory C. Reid. 2019. «Pornography problems due to moral incongruence: An integrative model with a systematic review and meta-analysis». Archives of Sexual Behavior, 48(2): 397-415.

Hald, Gert Martin y Alensandar Štulhofer. 2016. «What types of pornography do people use and do they cluster? Assessing types and categories of pornography consumption in a large-scale online sample». The Journal of Sex Research, 53(7): 849-859.

Nebot-Garcia, Juan Enrique, Marta García-Barba, Beatriz Gil-Juliá, Cristina GiménezGarcía y Rafael Ballester-Arnal. 2018. «Comportamientos homosexuales en jóvenes heterosexuales: diferencias de género». Revista Àgora de Salut, 5: 5968.

Nelson, Larry J., Laura M. Padilla-Walker y Jason S. Carroll. 2010. «"I believe it is wrong but I still do it": A comparison of religious young men who do versus do not use pornography». Psychology of Religion and Spirituality, 2(3): 136-147.

Park, Brian Y., Gary Wilson, Jonathan Berger, Matthew Christman, Bryn Reina, Frank Bishop, Warren P. Klam y Andrew P. Doan. 2016. «Is Internet pornography causing sexual dysfunctions? A review with clinical reports». Behavioral Sciences, 6(3): 17.

Poulsen, Franklin, Dean M. Busby y Adam M. Galovan. 2013. «Pornography use: Who uses it and how it is associated with couple outcomes». The Journal of Sex Research, 50(1): 72-83.

Priolo, Daniel, Isabelle Milhabet, Olivier Codou, Valérie Fointiat, Emmanuelle Lebarbenchon y Fabrice Gabarrot. 2016. «Encouraging ecological behaviour through induced hypocrisy and inconsistency». Journal of Environmental Psychology, 47: 166-180.

Rissel, Chris, Juliet Richters, Richard O. de Visser, Alan McKee, Anna Yeung y Theresa Caruana. 2017. "A profile of pornography users in Australia: Findings from the second Australian study of health and relationships». The Journal of Sex Research, 54(2): 227-240.

Ševčíková, Anna y Kristian Daneback. 2014. «Online pornography use in adolescence: Age and gender differences». European Journal of Developmental Psychology, 11(6): 674-686.

Tylka, Tracy L. 2015. «No harm in looking, right? Men's pornography consumption, body image, and well-being». Psychology of Men \& Masculinity, 16(1): 97-107.

Van Ness, Nicole, Marianne M. Miller, Sesen Negash y Martha Morgan. 2017. «Embracing our eroticism: a foucauldian discourse analysis of women's eroticism». Journal of Feminist Family Therapy, 29(3): 103-126.

Vrangalova, Zhana y Ritch C. Savin-Williams. 2010. "Correlates of same-sex sexuality in heterosexually identified young adults». The Journal of Sex Research, 47(1): 92-102. 
Walters, Nathan T. y Paul M. Spengler. 2016. «Clinical errors and therapist discomfort with client disclosure of troublesome pornography use: Implications for clinical practice and error reduction». Psychotherapy, 53(3): 354-359.

Willoughby, Brian J., Jason S. Carroll, Dean M. Busby y Cameron C. Brown. 2016. «Differences in pornography use among couples: Associations with satisfaction, stability, and relationship processes». Archives of Sexual Behavior, 45(1): 145158.

Wright, Paul J., Ekra Miezan y Chyng Sun. 2019. «Pornography Consumption and Sexual Satisfaction in a Korean Sample». Journal of Media Psychology: Theories, Methods, and Applications, 31(3): 164-169.

Yu, Calvin Kai-Ching. 2013. «Lust, pornography, and erotic dreams». Dreaming, 23(3): 175-193. 